\section{Experiencia en el Foro Global de Bioética en Investigación. Desafíos para la revisión ética en Chile}

\author{
SOFÍA P. SALAS
}

\section{Experience in the global forum of bioethics in research. Challenges for the ethical review in Chile}

The Global Forum on Bioethics in Research annually convenes a number of researchers, bioethicists and stakeholders with a shared interest in the ethics of conducting research in low and middle-income countries (LMIC). It provides a useful platform to discuss ethical issues that affect research practice in different scenarios, promoting ethically conducted research, global development for health research ethics and partnerships between the global north and south. As participant of the last three meetings, in this article the author analyzes the main ethical issues that were discussed in this forum, namely "Emerging epidemic infections and experimental medical treatments" (Annecy, France, 2015); "Ethics of research in pregnancy" (Buenos Aires, Argentina, 2016), and "The ethics of alternative clinical trial designs and methods in LMIC research" (Bangkok, Thailand, 2017). Local research ethics committees are not well prepared to face the new ethical challenges associated with research conducted in emergency situations or in pregnant women, or to evaluate new methods, such as alternative clinical trial designs (cluster randomized trials, adaptive platforms, or controlled human infection models, among others). According to this scenario, research ethics committees should be trained to carefully assess the risks and benefits of approving this type of research. In this context, it is necessary to harmonize local regulations with the new international standards in research ethics.

(Rev Med Chile 2018; 146: 653-659)

Key words: Clinical Protocols; Epidemiologic Research Design; Ethics; Ethics Committees; Informed Consent; Research.
${ }^{1}$ Centro de Bioética, Facultad de Medicina, Universidad del Desarrollo Clínica Alemana. Santiago, Chile.

Conflicto de interés: Ninguno que declarar.

Recibido el 19 de diciembre de 2017, aceptado el 29 de mayo de 2018.

Correspondencia a:

Sofía P. Salas

Centro de Bioética,

Facultad de Medicina, Clínica

Alemana Universidad del

Desarrollo

Avenida Las Condes 12.461,

Santiago, Chile.

Fono: +562- 23279157

sofiasalas@udd.cl
$\mathrm{E}$ 1 Foro Global de Bioética en Investigación (GFBR por sus siglas en inglés), tiene como objetivos promover que la investigación se realice de manera ética, fortalecer la ética de la investigación en salud y estimular la colaboración entre países ${ }^{1}$. Desde el año 1999, realiza reuniones periódicas que facilitan que investigadores y bioeticistas de distintos países expongan las dificultades que experimentan a nivel local para conducir de manera ética sus investigaciones. He tenido el privilegio de asistir a las tres últimas reuniones del GFBR, en las cuales se han discutido aspectos emergentes de ética en investigación que plantean desafíos adicionales para los Comités Ético Científicos (CEC). En el presente artículo, discutiré brevemente los principales temas abordados en estos foros y señalaré algunos desafíos para implementar estas propuestas en Chile. 


\section{Ética de la investigación en emergencia sanitaria}

El Foro realizado en Annecy, Francia, en noviembre de 2015, estuvo dedicado a los aspectos éticos relacionados con "Infecciones epidémicas emergentes y tratamientos médicos experimentales". Expertos internacionales discutieron la forma en que los países afectados por la epidemia de Ébola abordaron los desafíos éticos relacionados con realizar investigación durante epidemias, especialmente en un contexto donde no hay disponible terapia ${ }^{2}$. Existen desafíos éticos específicos para investigar en estas circunstancias, debido a la alta morbimortalidad asociada a la condición, la escasez de recursos disponibles en las regiones afectadas y la necesidad de tomar decisiones apresuradas, basadas en información incompleta y de validez incierta. Debido a los resultados de ensayos clínicos realizados en situación de emergencia, que incluyeron el uso de fármacos o vacunas que por primera vez se probaban en seres humanos, se dispuso de nuevos tratamientos y vacunas en un tiempo relativamente corto. Todo esto fue posible gracias a que se adecuaron los requisitos éticos para realizar investigación en emergencias graves (para mayor información, ver referencia $\left.{ }^{3}\right)$, los que armonizaron la necesaria celeridad para lograr aprobación ética con la debida protección de los sujetos de investigación. Para la aprobación ética de dichos estudios, realizados en población extremadamente vulnerable, se tuvo especial consideración al tema del consentimiento informado (CI), puesto que en esta situación de emergencia sanitaria, muchas veces se reclutaron a personas que no estaban en condiciones de otorgar un CI válido o, de estarlo, era casi imposible que los participantes no sintieran que cualquier terapia experimental que se les ofreciera era una "cura milagrosa”. La obtención de un CI válido, en condiciones de fragilidad extrema de los participantes, impone una dificultad adicional, puesto que necesariamente eran reclutados durante una estricta cuarentena sanitaria, era necesario recurrir a consentimiento por subrogación, debido a que el participante no estaba en condiciones de otorgarlo, y también habían niños infectados cuyos padres no eran habidos, pero para los cuales la alternativa de no participar en los ensayos significaba excluirlos de la única terapia disponible.

También se profundizó respecto de cuál debe ser el acceso a muestras y datos, cuál debe ser el acceso al tratamiento que se muestre como eficaz, y cuáles son las obligaciones posteriores al ensayo, aspectos que están estrechamente entrelazados con la voluntad de los países y las comunidades en participar en los esfuerzos locales e internacionales para realizar investigación durante situación de epidemias, especialmente cuando éstas implican riesgos severos de morbimortalidad ${ }^{2}$.

Como una manera de hacer frente a las dificultades metodológicas que plantea el realizar ensayos clínicos con terapias muchas veces nunca antes probadas en humanos, se consideró un "diseño de ensayo adaptativo", como alternativa al tradicional ensayo randomizado a un grupo control y otro con la nueva terapia. El principio de "equipoise" justifica la asignación al azar de algunos participantes al grupo placebo o atención estándar. Sin embargo, el conocimiento recolectado durante el ensayo no es binario sino en un espectro; en algún momento puede quedar claro que un tratamiento es superior a la atención estándar, lo que cambia el imperativo ético para proporcionar el tratamiento experimental a más participantes. En los diseños adaptativos, el ensayo se modifica sobre la base de lo que se aprende acerca de la intervención, permitiendo que un mayor porcentaje de pacientes reciba el tratamiento experimental que posiblemente es eficaz. Los participantes en el encuentro estuvieron de acuerdo en que esto no elimina la tensión ética que significa que sólo algunos sujetos reciben el nuevo tratamiento, pero sí la reduce significativamente. El tema de los diseños alternativos fue discutido en mayor profundidad en el encuentro realizado el 2017 (ver más adelante).

Es interesante señalar que las normas del Council of International Organizations of Medical Sciences (Pautas CIOMS), aprobadas el 2016, incluyen recomendaciones para la investigación en situaciones de desastre y brotes de enfermedades (Pauta $20^{4}$ ) y reconocen algunos desafíos específicos, tales como la "necesidad de generar conocimiento rápidamente, mantener la confianza pública y superar obstáculos prácticos a la ejecución de la investigación", aspectos que deben ser equilibrados con la necesidad de asegurar la validez científica de la investigación y con la mantención de los principios éticos en su ejecución. Los principales requisitos éticos que considera CIOMS para este tipo de investigación se describen en la Tabla 1. 
Tabla 1. Requisitos éticos para investigación en situación de desastre y brotes de enfermedades, según CIOMS 2016

\begin{tabular}{|c|c|c|}
\hline Pauta & Título & Requisitos \\
\hline Pauta 1 & Valor social y científico, y respeto de los derechos & $\begin{array}{l}\text { Diseño del estudio adecuado para producir resulta- } \\
\text { dos con validez científica }\end{array}$ \\
\hline Pauta 2 & Investigación en entornos de escasos recursos & $\begin{array}{l}\text { La investigación responde a las necesidades de salud } \\
\text { de las víctimas del desastre y no puede realizarse } \\
\text { fuera de esta situación }\end{array}$ \\
\hline Pauta 3 & $\begin{array}{l}\text { Distribución equitativa de beneficios y cargas en la } \\
\text { selección de individuos y grupos de participantes en } \\
\text { una investigación }\end{array}$ & $\begin{array}{l}\text { Selección justa de participantes y se justifica cuando } \\
\text { se escogen o excluyen a determinadas poblaciones; } \\
\text { existe distribución equitativa de las cargas y benefi- } \\
\text { cios de participar en una investigación }\end{array}$ \\
\hline Pauta 4 & $\begin{array}{l}\text { Posibles beneficios individuales y riesgos de una } \\
\text { investigación }\end{array}$ & $\begin{array}{l}\text { Evaluación realista de los riesgos y posibles bene- } \\
\text { ficios individuales de la intervención, sobretodo } \\
\text { cuando están en fase inicial }\end{array}$ \\
\hline Pauta 7 & Involucramiento de la comunidad & $\begin{array}{l}\text { Participación activa de la comunidad en la planifica- } \\
\text { ción del estudio, de tal modo que sea pertinente cul- } \\
\text { turalmente y que se afronten los desafíos prácticos }\end{array}$ \\
\hline Pautas 9 y 10 & $\begin{array}{l}\text { Personas que tienen capacidad de dar Cl y Modifica- } \\
\text { ciones y dispensas del } \mathrm{Cl}\end{array}$ & $\begin{array}{l}\text { Obtención del } \mathrm{Cl} \text { individual de los participantes, a } \\
\text { menos que existan las condiciones de dispensa del } \mathrm{Cl}\end{array}$ \\
\hline Pautas 2 y 23 & $\begin{array}{l}\text { Investigación en entornos de escasos recursos y } \\
\text { Requisitos para establecer comités de ética de la } \\
\text { investigación y para la revisión de protocolos }\end{array}$ & $\begin{array}{l}\text { Existe obligación ética de compartir los resultados } \\
\text { de la investigación y cualquier intervención efectiva } \\
\text { o nuevo conocimiento se pone a disposición de las } \\
\text { comunidades afectadas }\end{array}$ \\
\hline
\end{tabular}

Adaptada de Referencia 4. Cl = Consentimiento Informado.

\section{Aspectos éticos de la investigación en embarazadas}

En el foro realizado el 2016 en Buenos Aires, Argentina, se discutieron los aspectos éticos de la investigación en embarazadas, especialmente en el contexto de la epidemia de virus Zika que afectaba a Latinoamérica ${ }^{5}$. Si bien la mayoría de los países no tiene regulación que restrinja la investigación durante el embarazo y/o la lactancia, la evidencia muestra que los investigadores y los CEC consideran que las mujeres embarazadas deben ser excluidas de participar como sujetos de investigación, principalmente por los potenciales riesgos fetales. Esto ha significado que una minoría de fármacos ha sido testeado para determinar su eficacia y seguridad durante el embarazo, a pesar de que las embarazadas requieren de tratamientos validados para su uso en condiciones pre-existentes y también para enfermedades específicas de la gestación, puesto que las mujeres con enfermedades se embarazan y las embarazadas se enferman ${ }^{5}$. Durante la reunión se originó consenso respecto de que la investigación en embarazadas era moralmente relevante, puesto que era fundamental poder entregar a las mujeres medidas preventivas y terapéuticas que fuesen seguras y eficaces, tanto para ellas como para el feto. A partir de esta discusión, el grupo de participantes de Latino América y El Caribe hizo un llamado ético para la inclusión de mujeres embarazadas en investigación, de manera responsable ${ }^{1}$.

Los temas de mayor interés en la discusión fueron el concepto de vulnerabilidad, balance riesgo/beneficio, consentimiento informado, aspectos regulatorios y la investigación en embarazadas en una emergencia sanitaria. En relación al concepto de vulnerabilidad, hubo acuerdo que la embarazada no era "vulnerable", a menos que tuviese alguna circunstancia particular que la hiciese particularmente susceptible a ser dañada por la investigación, en línea con las normas CIOMS ${ }^{4}$. Se consideró a este grupo como "población especial", para permitir su participación en investigación, teniendo en consideración las necesidades especiales que pudiesen tener. 
Respecto del balance riesgo/beneficio, se estimó pertinente que éste fuese determinado de manera específica para cada potencial participante, sin que fuese necesario que hubiese un beneficio directo para la madre o el feto. En este aspecto, se concluyó que era relevante que los CEC evaluaran el riesgo de participar y el riesgo de no hacerlo, tanto para la madre como para el feto; a su vez, los investigadores deben justificar por qué las embarazadas deben ser excluidas del estudio si existe la posibilidad de que la investigación pueda beneficiarla a ella, a su feto, o a las embarazadas en general. Esto implica un cambio de paradigma, desde la exclusión sistemática al de inclusión, a menos que se establezca un balance riesgo/beneficio desfavorable.

En relación al CI, se consideró que lo ideal era obtenerlo en forma precoz, antes incluso que se presente la condición; esta estrategia permitiría que la mujer tome decisiones sin la presión de resolver enfrentada a una situación de emergencia $^{5,6}$; se recalcó que el consentimiento definitivo para participar o denegar la participación debiese ser otorgado por la embarazada y no por terceros.

Hubo consenso que se necesitaba una estandarización de conceptos que no están bien definidos, como por ejemplo, qué se entiende por riesgo mínimo, o cómo resolver situaciones en las cuales las leyes vigentes entran en conflicto con las posibilidades de participar en investigación. Un ejemplo de esto es la posibilidad de considerar a las menores embarazadas como capaces de consentir en la investigación, a menos que se demuestre que no tienen capacidad para hacerlo. Nuevamente surgió la necesidad de que los CEC locales se capaciten en entender que la investigación en mujeres embarazadas sí se puede realizar de manera responsable, tal como lo señala CIOMS (Pauta 194). Las tres áreas específicas en las que CIOMS promueve que se realicen investigaciones en mujeres durante el embarazo y lactancia, se muestran en la Tabla 2.

Finalmente, en pleno brote de la epidemia de Zika, se discutió extensamente si era ético o no incluir a embarazadas en ensayos clínicos con vacunas que nunca antes habían sido probadas en humanos. Dado que los principales afectados por la infección eran la embarazada y su feto se consideró que era poco ético excluirlas de una investigación que potencialmente podría mejorar el riesgo de microcefalia. Al respecto, si una investigación cuenta con la aprobación de un CEC el cual ha determinado que el mejor beneficio para la embarazada es participar en un ensayo clínico en situación de emergencia, esta opción debe ser ofrecida para que ella tome una decisión informada. Considerando que es muy probable que sigan existiendo brotes infecciosos que afecten a embarazadas, los requisitos para ser incluidas o excluidas en los futuros ensayos deben ser acordados con las autoridades regulatorias y los patrocinadores antes de una nueva epidemia. Un aspecto adicional, que también fue considerado en la revisión de las guías CIOMS, fue que la investigación en embarazadas solo debe realizarse en entornos donde pueda garantizarse el acceso a un aborto seguro y legal para las mujeres que así lo deseen, con el objeto de impedir que den a luz un niño malformado en contra de sus deseos (CIOMS, Pauta $\left.19^{4}\right)$.

\section{Ensayos clínicos alternativos}

En el foro realizado en Bangkok, Tailandia, en noviembre de 2017, se discutió la "Ética de

\section{Tabla 2. Áreas en las cuales debe realizarse investigaciones en mujeres embarazadas y lactantes,} según CIOMS 2016

\begin{tabular}{|ll}
\hline Áreas & Ejemplos \\
\hline $\begin{array}{l}\text { Intervenciones para condiciones específicas resultantes del } \\
\text { embarazo }\end{array}$ & $\begin{array}{l}\text { Aquellas relacionadas con el tratamiento de la pre- } \\
\text { eclampsia-eclampsia }\end{array}$ \\
$\begin{array}{ll}\text { Intervenciones para condiciones que afectan a la población } \\
\text { general y que razonablemente se podría esperar que se usen sin }\end{array}$ & $\begin{array}{l}\text { Empleo de medicamentos para tratar condiciones preva- } \\
\text { levidencia adecuada durante el embarazo y lactancia }\end{array}$ \\
zación para su uso en embarazo o lactancia
\end{tabular}

Adaptada de Referencia 4. 
los diseños y métodos alternativos de ensayos clínicos". Como mencionamos antes, para hacer frente a la epidemia de Ébola fue necesario realizar adecuaciones a los tradicionales ensayos clínicos con el objeto de acortar los tiempos requeridos para encontrar evidencia científica válida. Entre los modelos discutidos estuvieron los ensayos aleatorizados por conglomerado (EAC, cluster randomized trials), los ensayos con plataformas adaptativas (adaptive platforms) y los de infecciones controladas en seres humanos (CHIMs, de sus siglas en inglés). Cada uno de estos modelos provee dificultades éticas que le son particulares, pero por motivos de espacio me referiré sólo al primero. Los EAC son cada vez más importantes para evaluar una intervención en salud. Según este diseño, los grupos o conglomerados de individuos son randomizados a las ramas de estudio, para luego medir los resultados en forma individual en cada sujeto perteneciente al conglomerado. El principal desafío ético es que las unidades de randomización, de intervención y de obtención de resultados son distintas, lo que dificulta la obtención del $\mathrm{CI}^{7}$. En algunos EAC, las intervenciones se administran a nivel del conglomerado, por lo que afectan directa o indirectamente los intereses de muchos individuos, incluyendo a visitantes de la comunidad intervenida. Por otra parte, en este tipo de diseños es frecuente que la randomización se realice antes que se pueda identificar y reclutar a los individuos para que den su CI, por lo que no es factible obtenerlo previo a la randomización. Asimismo, las intervenciones pueden ser muy difíciles de evitar por los sujetos expuestos, lo que dificulta el rechazar la intervención; hay dudas respecto del papel del líder de la comunidad que entrega el acceso a ésta y también hay dificultades en evaluar el balance riesgo/beneficio a nivel individual respecto de aquél que puede experimentar el conglomerado en su conjunto. Todos estos son aspectos novedosos, que deben ser abordados en profundidad por los CEC al evaluar estos protocolos. Un grupo de expertos internacionales elaboró una declaración en la cual realizan 15 recomendaciones para aplicar los principios éticos a estos nuevos diseños ${ }^{7}$. En síntesis, estas recomendaciones hacen referencia a la necesidad de justificar de manera adecuada este tipo de diseño, la necesidad de revisión por parte de un CEC, la identificación de los sujetos de estudio, la obtención del consentimiento infor-

\section{Tabla 3. Consideraciones éticas para realización de ensayos aleatorizados por conglomerado (EAC),} según CIOMS 2016

\section{Consideraciones éticas}

Determinar quiénes son los participantes en la investigación y quiénes más se ven afectados directa o indirectamente

Determinar si se requiere y es factible obtener el $\mathrm{Cl}$ de pacientes, trabajadores de la salud o miembros de la comunidad, según el tipo de estudio

Determinar si el requerir el $\mathrm{Cl}$ o permitir denegar el $\mathrm{Cl}$ puede invalidar los resultados de la investigación

Determinar si es ético tener un conglomerado sin intervención como comparador en un ensayo aleatorizado por conglomerado

Decidir si se requiere autorización de la persona que controla el acceso al conglomerado

\section{Argumentos y ejemplos}

Introducción de un nuevo procedimiento para controlar infecciones en los trabajadores de la salud en los cuales se registran datos consolidados del número de infecciones. Los pacientes no son sujetos de estudio, pero se necesitan sus datos consolidados

En ocasiones se hace imposible obtener un $\mathrm{Cl}$ individual, puesto que la intervención se dirige a toda una comunidad; o es necesario que el grupo control desconozca la intervención, para evitar cambios de comportamiento

Cuando el estudio se realiza a nivel de conglomerado (ie. Hospitales o comunidades), el requisito de $\mathrm{Cl}$ para los trabajadores de la salud puede comprometer los resultados de la investigación, porque si rehúsan participar, se podrían alterar los resultados de la intervención

Cuando la intervención ha sido probada como eficaz (por lo tanto, es una investigación sobre implementación), no sería ético privar de la intervención al grupo control si es que esto expone a los participantes a un riesgo mayor que el mínimo

Si el EAC afecta los intereses del conglomerado u organización, puede ser conveniente obtener la autorización del líder comunitario o directivo de la agrupación ("gatekeeper"); esto no reemplaza el Cl individual

Adaptada de Referencia 4. $\mathrm{Cl}=$ Consentimiento Informado. EAC = ensayos aleatorizados por conglomerado. 
mado, el rol del representante de la comunidad, la determinación de los beneficios y riesgos y la protección de los sujetos vulnerables.

\section{Desafíos para la revisión ética en Chile}

La participación en estos encuentros internacionales, donde expertos debatieron sobre los cursos de acción más éticos para enfrentar nuevos desafíos en investigación, obliga a mirar lo que estamos haciendo en Chile. Con especial preocupación vemos la falta de capacidades en el sistema de revisión ética local para balancear el interés de protección de los sujetos de investigación con el interés en el avance de la investigación en necesidades de salud del país, especialmente en contextos de emergencia sanitaria. Ese déficit afecta a varios actores del sistema, entre ellos los CEC y las autoridades sanitarias y regulatorias. A modo de ejemplo, un reciente documento de la división jurídica del MINSAL motivado por la consulta de un CEC en la revisión de un protocolo en contexto de emergencia sanitaria, ha considerado que el representante legal del potencial sujeto de investigación debe estar constituido por medio de sentencia judicial, acreditándose la representación invocada, por medio de "sentencia judicial firme y ejecutoriada...." un requerimiento inédito para investigación en sujetos que no pueden consentir. Al respecto, la ley de investigación (Ley 20.120) considera que el CI puede ser otorgado por el sujeto de investigación "o en su defecto, el de aquel que deba suplir su voluntad en conformidad con la ley" . Asimismo, la historia de esta Ley determina que si “... se trate de una situación de urgencia para la persona, será considerado representante quien a cuyo cuidado la persona se encuentre ...."10

\section{Conclusiones}

Compartiendo el juicio de otros autores, la participación en investigación es una obligación moral por razones de justicia, beneficencia y también por auto-desarrollo. Puesto que todos nos beneficiamos de los avances de la medicina moderna, todos somos igualmente requeridos para hacer nuestra contribución al avance del conocimiento médico ${ }^{11}$. Otros han considerado que la investigación biomédica es un bien público y, como tal, todos debemos contribuir a ella ${ }^{12}$. Para que esta contribución al desarrollo de la ciencia y la salud humana se realice de manera ética, aparece como indispensable que los CEC se capaciten en los desafíos que implican estos nuevos protocolos, evaluando de manera cuidadosa los riesgos y beneficios de participar en la investigación, y también los riesgos y beneficios de no hacerlo, para así flexibilizar la forma de llevar a cabo los protocolos de investigación ante emergencias epidemiológicas o naturales, con diseños que se adapten a las realidades particulares de cada país, tal como propusimos en nuestro anterior trabajo ${ }^{3}$.

Agradecimientos: Mi participación en los tres GFBR ha sido posible gracias a fondos provenientes del Wellcome Trust, de los Institutos Nacionales de la Salud de los Estados Unidos (NIH, por sus siglas en inglés), del Consejo de Investigación Médica del Reino Unido y de la Fundación Bill y Melinda Gates. La autora agradece al Profesor Alberto Lecaros Urzúa, por la revisión crítica de este manuscrito.

\section{Referencias}

1. Saenz C, Alger J, Beca JP, Belizan JM, Cafferata ML, Guzman JAC, et al. Un llamado ético a la inclusión de mujeres embarazadas en investigación. Rev Panam Salud Publica 2017; 41: e13.

2. Global Forum on Bioethics in Research. Reporte de la reunión GFBR-2015 Emerging epidemic infections and experimental medical treatments. Disponible en http:// www.gfbr.global/past-meetings/10th-forum-annecy-france-3-4-november-2015/. (Fecha de acceso el 14 de diciembre de 2017).

3. Beca JP, Salas SP. Problemas éticos y de salud planteados por la reciente epidemia de Ébola: ¿Qué debemos aprender?. Rev Med Chile 2016; 144 (3): 371-6.

4. International ethical guidelines for health-related research involving humans. Geneva, Switzerland: Council for International Organizations of Medical Sciences (CIOMS), 2016.

5. Global Forum on Bioethics in Research. Reporte de la reunión GFBR-2016 Ethics of research in pregnancy. Disponible en http://www.gfbr.global/past-meetings/ 11 th-forum-buenos-aires-argentina-3-4-november-2016/. (Fecha de acceso el 15 de diciembre de 2016).

6. Salas SP. Ethical challenges posed by clinical trials in 
preterm labor: a case study. Reproductive Health 2017; 14 (Suppl 3): 35-8.

7. Weijer C, Grimshaw JM, Eccles MP, McRae AD, White A, Brehaut JC, et al. The Ottawa Statement on the Ethical Design and Conduct of Cluster Randomized Trials. PLoS Med 2012; 9 (11): e1001346.

8. ORD. A15 No 4258 del 8 noviembre 2017, referido a interpretación de la Ley No 20.120 y su reglamento. Ministerio de Salud, Subsecretaría de Salud Pública, División Jurídica.

9. Ley No 20.120. Sobre la investigación científica en el ser humano, su genoma, y prohibe la clonación humana. Ministerio de Salud de Chile, promulgada el 7 septiem- bre 2006. Disponible en http://bcn.cl/1m19j. (Fecha de consulta el 18 de diciembre de 2017).

10. Historia de la Ley № 20.120 Sobre investigación científica en el ser humano, su genoma, y prohíbe la clonación humana. Ministerio de Salud de Chile, 22 de septiembre 2006. Disponible en http://www.bcn.cl/obtienearchivo?id=recursoslegales $/ 10221.3 / 566 / 1 / H L 20120$.pdf. (Fecha de consulta el 18 de diciembre de 2017).

11. Rhodes R. Rethinking research ethics. Am J Bioeth 2005; 5 (1): 7-28.

12. Schaefer GO, Emanuel EJ, Wertheimer A. The obligation to participate in biomedical research. JAMA 2009; 302 (1): 67-72. 